\title{
Outcomes of Non-Variceal Upper Gastrointestinal Bleed Stratified by Hospital Teaching Status: Insights From the National Inpatient Sample
}

\author{
Jennifer C. Asotibe ${ }^{\mathrm{a}, \mathrm{c}}$ (), Hafeez Shaka ${ }^{\mathrm{a}}$, Emmanuel Akuna ${ }^{\mathrm{a}}$, Niveda Shekara ${ }^{\mathrm{a}}$, Hassam Shah ${ }^{\mathrm{a}}$, \\ Marcelo Ramirez ${ }^{a}$, Syed Ali Amir Sherazia ${ }^{a}$ Katayoun Khoshbina, \\ Hemant Mutneja ${ }^{\mathrm{b}}$, Bashar Attar ${ }^{\mathrm{b}}$
}

\begin{abstract}
Background: Non-variceal upper gastrointestinal bleeding (NVUGIB) is a significant cause of mortality and morbidity in the USA. Currently, there are limited data on the inpatient outcomes of patients admitted with a diagnosis of NVUGIB stratified according to teaching hospital status. We analyzed data from the National Inpatient Sample (NIS) intending to evaluate these outcomes.
\end{abstract}

Methods: We queried the NIS 2016 and 2017 databases for NVUGIB hospitalizations by teaching hospital status. The primary outcome was inpatient mortality while secondary outcomes were rate of endoscopy for hemostasis, rate of early endoscopy (endoscopy in 1 day or less), mean time to endoscopy, rate of complications including acute kidney injury (AKI), acute respiratory failure (ARF), need for blood transfusion, development of sepsis, need for endotracheal intubation and mechanical ventilation as well as healthcare utilization.

Results: There were over 71 million weighted discharges in the combined 2016 and 2017 NIS database. A total of 94,900 NVUGIB cases were identified with $63.4 \%$ admitted in teaching hospitals. The in-hospital mortality for patients admitted with an NVUGIB in teaching hospitals was $1.98 \%$ compared to $1.5 \%$ in non-teaching hospitals (adjusted odds ratio (aOR): $1.38,95 \%$ confidence interval $(\mathrm{CI}): 1.08-1.77, \mathrm{P}=$ 0.010 ) when adjusted for biodemographic and hospital characteristics as well as comorbidities. Patients admitted with a diagnosis of NVUGIB in teaching hospitals had a 10\% adjusted increased odds of getting endoscopy for hemostasis $(27.0 \%$ vs. $24.5 \%$, aOR: $1.10,95 \%$ CI: $1.02-1.19$, $\mathrm{P}=0.016$ ) compared to patients in non-teaching hospitals. There was, however, no difference in early endoscopy between the two groups.

Manuscript submitted June 18, 2021, accepted July 29, 2021

Published online September 9, 2021

a Department of Internal Medicine, John H Stroger Hospital of Cook County, Chicago, IL, USA

bDepartment of Gastroenterology, John H Stroger Hospital of Cook County, Chicago, IL, USA

${ }^{\mathrm{c} C}$ Corresponding Author: Jennifer C. Asotibe, Department of Internal Medicine, John H Stroger, Jr Hospital of Cook County, 1969 W Ogden Avenue, Chicago, IL 60612, USA. Email: Jenniferasotibe@yahoo.com.sg

doi: https://doi.org/10.14740/gr1437
Conclusion: Patients admitted at teaching hospitals for an NVUGIB had worse outcomes during hospitalizations including mortality, median length of stay, and total hospital charges when compared to NVUGIB patients managed at non-teaching hospitals.

Keywords: Non-variceal upper gastrointestinal bleeding; Teaching hospital; Non-teaching hospital; Length of stay; Mortality; Total hospital charges

\section{Introduction}

Upper gastrointestinal bleeding (UGIB) is defined as GI hemorrhage from the oral cavity to the ligament of Treitz [1]. While an estimated $10-20 \%$ of UGIB episodes are usually secondary to portal hypertension, the majority of UGIB causes are usually non-variceal $[1,2]$. Non-variceal upper gastrointestinal bleeding (NVUGIB) remains a common clinical problem in the USA with an estimated annual incidence of 67 per 100,000 individuals [3]. With the advent of novel pharmacologic and endoscopic techniques for homeostasis, the incidence and mortality of UVUGIB have steadily decreased over the last two decades. However, mortality did not follow this trend and remained with a range of 1-5\% [3]. Amongst hospitalized patients with severe comorbidities, the mortality range may even reach $15 \%$ [4].

Multiple studies have reported disparities in healthcare and patient safety outcomes when comparing care delivery at teaching vs. non-teaching hospitals [5-7]. While earlier studies have reported a higher quality of care at teaching hospitals, other studies have reported no difference in outcomes in teaching hospitals [5-9]. For example, while Burke et al reported lower mortality rates for common conditions in patients managed at teaching hospitals when compared to non-teaching hospitals [10], Papinacolau et al and Au et al reported no differences in outcomes $[6,7]$. It is also well known that teaching hospitals act as leaders in the education of residents and students [5]. With the implementation of work-hour regulations in residency programs, some studies have reported a decrease in short-term mortality among high-risk patients in teaching hospitals managed for common internal medicine conditions; however, data for UGIB are limited $[5,8]$. 
While Patel et al recently evaluated the outcomes of variceal upper GI bleeding in patients stratified by hospital teaching status and reported worse outcomes in teaching hospitals [5], no other study has compared the outcomes of NVUGIB between teaching and non-teaching hospitals. This lack of outcome data on NVUGIB amongst teaching and non-teaching hospitals prompted this research. We aimed to compare outcomes in teaching and non-teaching hospital settings at a national level.

\section{Materials and Methods}

\section{Data source and study population}

We conducted a retrospective cohort study of hospitalizations in 2016 and 2017 with a principal diagnosis of NVUGIB which included acute peptic ulcer, site unspecified with hemorrhage, ulcer of the esophagus with bleeding, acute duodenal ulcer with hemorrhage, angiodysplasia of stomach and duodenum with bleeding, Dieulafoy lesion (hemorrhagic) of stomach and duodenum, Mallory-Weiss syndrome with hemorrhage, and acute gastritis with bleeding. We used ICD-10 codes obtained from literature review of similar validated studies done on NVUGIB [11-13].

Data were sourced from the Nationwide Inpatient Sample (NIS) database for 2016 and 2017. The NIS is a database of hospital inpatient stays derived from billing data submitted by hospitals to statewide data organizations across the USA, covering more than $97 \%$ of the US population [14]. Each year approximates a $20 \%$ stratified sample of discharges from US community hospitals, excluding rehabilitation and long-term acute care hospitals. This dataset is weighted to obtain national estimates. Both the 2016 and 2017 databases are entirely coded using the International Classification of Diseases, Tenth Revision, Clinical Modification/Procedure Coding System (ICD-10-CM/PCS). In the NIS, diagnoses are divided into one principal diagnosis and secondary diagnosis. A principal diagnosis was the main ICD-10 code for the hospitalization. Secondary diagnoses were any ICD-10 code other than the principal diagnosis.

This manuscript is exempt from IRB approval as it involves data without patient identifiers. The data used in this study are readily available online at https://www.hcup-us.ahrq. gov. Ethical compliance with human/animal studies is not applicable as there were no animals used in this study and the study involves data without patient identifiers.

\section{Inclusion criteria and study variables}

The study population consisted of all hospitalizations for NVUGIB in the NIS 2016 and 2017. Study variables included sociodemographic and hospital characteristics including age, race, gender, primary payer, mean household income by quartile, disposition, hospital bed size, hospital location, and hospital teaching status which were included in the database. We used ICD-10 codes to obtain the comorbidities mapped from Deyo's adaptation of the widely validated Charlson Comorbidity Index (CCI) mentioned in the Supplementary Material 1 (www.gastrores.org) [15]. We excluded patients less than 18 years and elective hospitalization.

\section{Outcomes measures}

The primary outcome was comparing inpatient mortality from NVUGIB between teaching and non-teaching hospitals. Secondary outcomes studied included rate of endoscopy for hemostasis, rate of early endoscopy (endoscopy in 1 day or less), mean time to endoscopy, rate of complications including acute kidney injury (AKI), acute respiratory failure (ARF), need for blood transfusion, development of sepsis, need for endotracheal intubation and mechanical ventilation. We also compared the mean length of stay (LOS) and the mean total hospital charges (THC) between teaching and non-teaching hospitals as a surrogate marker for healthcare cost utilization.

\section{Statistical analysis}

We analyzed the data using Stata ${ }^{\circledR}$ Version 16 software (StataCorp, Texas, USA). We conducted all the analysis using the weighted samples for national estimates in adjunct with Healthcare Cost and Utilization Project (HCUP) regulations for using the NIS databases. Age grouped as 18 - 39 years representing young adults, 40 - 64 years representing middle-aged adults, and 65 years above representing elderly. We calculated comorbidities as proportions of the cohorts and used the Chisquare test to compare characteristics between the index and readmissions. We used univariable regression to compare both primary and secondary outcomes to obtain adjusted odds ratios (aORs). We carried out a backward stepwise multivariable regression analysis on sex, age categories, race, primary payer, household income quartiles, hospital bed size, hospital location, and 17 comorbidities that make up the CCI, which is similar to the model employed by Moore et al for assessing comorbidity burden in administrative databases [16]. Selection involved removing variables with $\mathrm{P} \geq 0.2$ and adding variables with $\mathrm{P}<0.1$ to create the final model for readmissions. The final model included age categories, sex, race, human immunodeficiency virus/acquired immunodeficiency syndrome (HIV/ AIDS), moderate or severe liver disease, metastatic solid tumor, myocardial infarction, congestive heart failure, cerebrovascular disease, paraplegia, mild liver disease, chronic pulmonary disease, rheumatologic disease, peptic ulcer disease, and renal disease.

\section{Ethical considerations}

The NIS lacks patient identifiers. In keeping with other HCUP databases, the NIS does not require Cook County Health Institutional Review Board approval for analysis.

\section{Results}

\section{Patient and hospital characteristics}

There was a total of 94,900 hospitalizations for NVUGIB, of 
which $60,175(63.4 \%)$ were in teaching hospitals. Patients in teaching hospitals were significantly younger (mean age 62.8 vs. 64.8 years, $\mathrm{P}<0.001)$ and involved a lower proportion of women compared to non-teaching hospitals. Teaching hospitals had higher proportions of non-white patients and patients with private insurance. A larger proportion of hospitalizations in the teaching hospitals had a median income greater than the 50th centile compared to non-teaching hospitals.

Comorbidity distribution between both settings was varied. Patients in non-teaching centers were more likely to have comorbid dementia $(9.3 \%$ vs. $7.5 \%, \mathrm{P}<0.001)$, peptic ulcer disease $(28.6 \%$ vs. $26.7 \%, \mathrm{P}=0.009)$, chronic pulmonary disease, and diabetes without complications, while patients in teaching centers had a higher proportion of HIV $(0.5 \%$ vs. $0.2 \%, \mathrm{P}=0.009)$, renal disease $(22.4$ vs. $20.3, \mathrm{P}=0.001)$ and diabetes with chronic complications.

There was also significant regional and size distribution relative to teaching status as shown in Table 1.

\section{Outcomes}

Primary outcome: in-hospital mortality

The in-hospital mortality for patients admitted with an NVUGIB in teaching hospitals was $1.98 \%$ compared to $1.5 \%$ in non-teaching hospitals (aOR: 1.38, 95\% CI: $1.08-1.77, \mathrm{P}=$ 0.010 ) when adjusted for biodemographic and hospital characteristics as well as comorbidities. Early endoscopy was associated with a significantly lower odds of mortality (aOR: 0.66, 95\% CI: $0.45-0.97, \mathrm{P}=0.033$ ) adjusted for confounders.

\section{Secondary outcomes}

Patients admitted with a diagnosis of NVUGIB in teaching hospitals had a $10 \%$ adjusted increased odds of getting endoscopy for hemostasis $(27.0 \%$ vs. $24.5 \%$, aOR: $1.10,95 \%$ CI: $1.02-$ $1.19, \mathrm{P}=0.016$ ) compared to patients in non-teaching hospitals. There was, however, no difference in early endoscopy between the two groups. Teaching hospitals had higher odds of complications including the need for mechanical ventilation $(6.4 \%$ vs. 3.9\%, aOR: 1.62, 95\% CI: $1.38-1.90)$, and development of AKI (21.9 vs. 18.8, aOR: 1.17, 95\% CI: 1.07 - 1.28, $\mathrm{P}<0.001)$. Teaching hospitals had a higher mean LOS and mean THC when compared to patients admitted with the same diagnosis in non-teaching hospitals as detailed in Table 2.

\section{Discussion}

Utilizing a nationwide database, we aimed to compare mortality and other outcomes such as time to endoscopy, odds of developing renal failure, respiratory failure, sepsis, need for transfusion, mechanical intubation/ventilation, THC and LOS for NVUGIB among teaching versus non-teaching hospitals in the USA. Among 94,900 patients with NVUGIB hospitalizations, the median LOS, THC, and mortality were higher in teaching versus non-teaching hospitals. The odds of requiring intubation with subsequent mechanical ventilation, developing acute renal failure and ARF were also found to be subsequently higher in teaching hospitals. Teaching hospitals are widely reputed for a higher quality of care delivery, specialized services, advanced technologies, and innovative research [17]. Previous studies have found that risk-adjusted mortality and LOS were lower for patients admitted in major teaching hospitals than for patients in minor, and non-teaching hospitals $[10,18]$. In a recent study, it was found that amongst 589,180 individuals admitted for gastrointestinal bleeding, the 30-day mortality was $6.3 \%$ vs. $7.0 \%$ in major vs. minor teaching hospitals, respectively [10]. Factors such as hospital volume, early technology adaptation, and even teaching intensity were associated with higher performance and lower mortality among teaching hospitals $[10,19]$. In our study, we discovered worse overall outcomes at teaching hospitals.

Our study demonstrates that in-hospital mortality, odds of developing acute renal failure/respiratory failure, requiring intubation with subsequent mechanical ventilation, LOS, and THC were higher in patients admitted to teaching hospitals for NVUGIB compared to non-teaching hospitals. We attribute these findings primarily to the acuity of cases seen in primary academic medical centers. A study looking at the acuity among emergency departments found that the median case acuity in teaching hospitals not only was higher but also fell outside the interquartile range of non-teaching hospitals [20]. It makes sense then that since sicker patients are admitted to teaching hospitals, the overall inpatient outcomes would also be worse. The acuity of the cases managed at teaching hospitals probably impacts the mortality and in turn leads to extended LOS and higher THC.

Teaching hospitals have also been reported to rely heavily on government, Medicare, and Medicaid subsidies for both graduate medical education and patient reimbursement for revenue generation [21-23]. They are also known to serve the poor, underserved populations in the USA, which operate at a lower financial margin when compared to non-teaching hospitals [19]. It is well known that hospitals that are well financed are more equipped to improve and provide better patient quality care $[21,24,25]$. This may account for worse outcomes observed in teaching hospitals when compared to non-teaching hospitals in our study.

In our study, we found that patients managed in teaching hospitals had higher odds of obtaining an esophagogastroduodenoscopy (EGD) (27\% vs. $24.5 \%)$ when compared to patients managed at non-teaching hospitals. Our findings are echoed by studies that report that patients managed at teaching patients managed at teaching hospitals were more likely to get an EGD when compared to patients managed at non-teaching hospitals [5]. Both healthcare facilities were however found to have a similar time to endoscopy ( $<1$ day). It is interesting that although patients managed at teaching hospitals were more likely to obtain an EGD, patients at teaching hospitals still had worse overall outcomes. We hypothesize that these outcomes may pertain to the technical expertise required in performing endoscopic procedures. Indeed, EGD and therapeutic endoscopy interventions are crucial in the successful management of patients with NVUGIB. The less desirable 
Table 1. Patient and Hospital Characteristics of Hospitalizations With NVUGIB by Hospital Setting

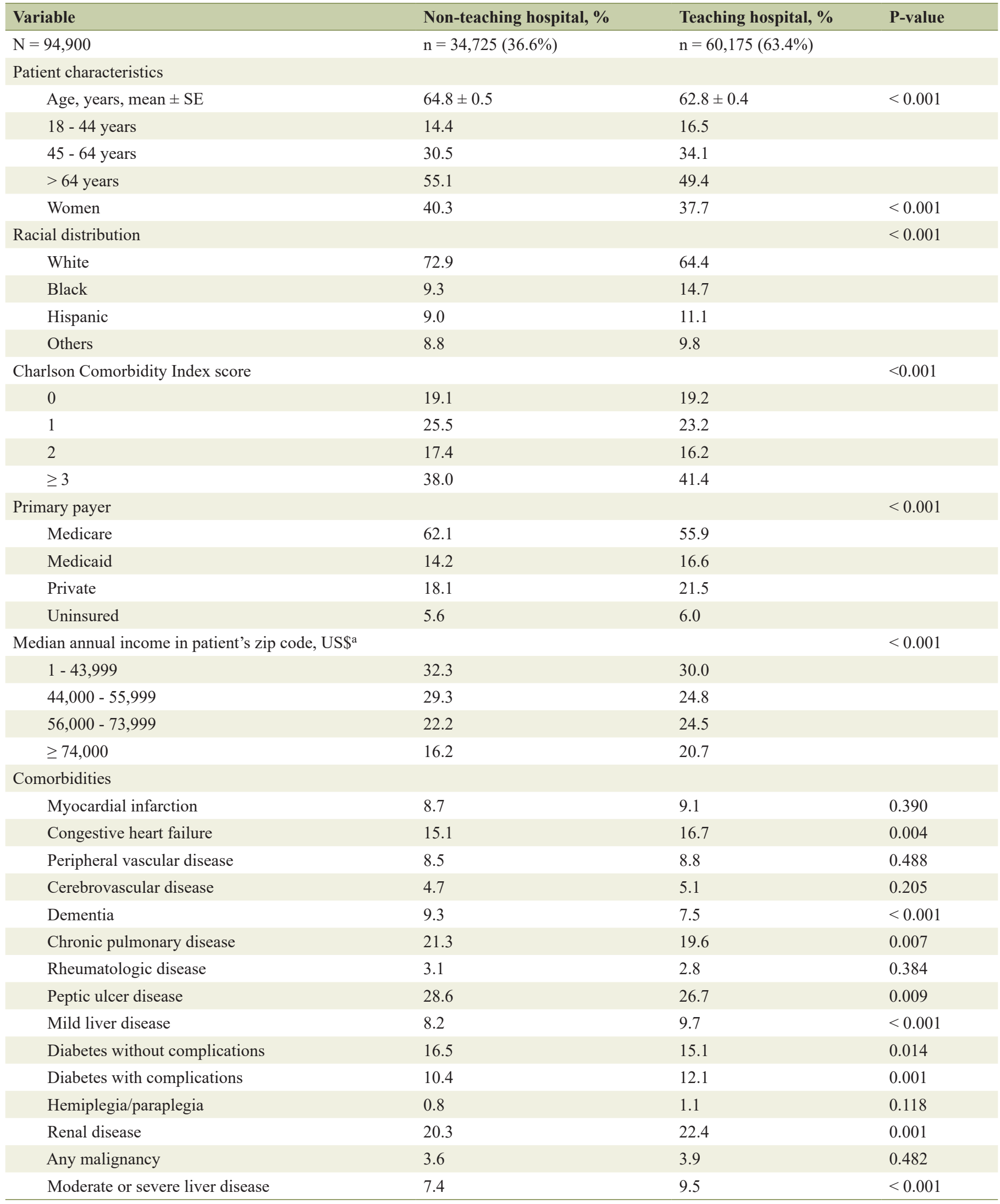


Table 1. Patient and Hospital Characteristics of Hospitalizations With NVUGIB by Hospital Setting - (continued)

\begin{tabular}{|clll}
\hline Variable & Non-teaching hospital, $\%$ & Teaching hospital, \% & P-value \\
\hline Metastatic solid tumor & 2.0 & 2.5 & 0.036 \\
HIV/AIDS & 0.2 & & \\
Hospital characteristics & & & \\
Hospital region & & 20.3 & \\
Northeast & 11.7 & 21.6 \\
Midwest & 19.7 & 36.3 \\
South & 42.4 & 21.8 \\
West & 26.2 & \\
Hospital bed size & & 23.0 \\
Small & 12.8 & 32.5 \\
Medium & 28.9 & 44.5 \\
Large & 58.3 & $<001$ \\
\hline
\end{tabular}

aFor 2017. NVUGIB: non-variceal upper gastrointestinal bleeding; SE: standard error of the mean; HIV: human immunodeficiency virus; AIDS: acquired immunodeficiency syndrome.

outcomes of patients with NVUGIB demonstrated by teaching hospitals in our study may be attributed in part to the level of competencies attained by the gastroenterology trainees in teaching hospitals. It has been reported that medical trainees may have a higher risk of being involved in medical errors [26]. This vulnerability may be related to their level of endoscopic skills, inexperience in handling unusual endoscopic presentations, level of attending physician supervision, and lack of hand-off mechanism between the primary GI service and the on-call GI service. These issues tend to be more challenging from July through September each year when the GI fellows begin their training. So although patients at teaching hospitals had more endoscopies, and both centers had similar times to early EGD, the level of competencies may have had an effect observed in our cohort of patients.

Finally, in our study, NVUGIB patients managed at teaching and non-teaching hospitals were found to have no statistically significant differences in the need for red blood cells (RBC) transfusion between the two groups. Patients managed at teaching and non-teaching hospitals had no difference in their time to endoscopy as mentioned above. Since endoscopy is vital to management to NVUGIB, it is understandable that

Table 2. Outcomes of NVUGIB Patients Managed at Non-Teaching vs. Teaching Hospitals

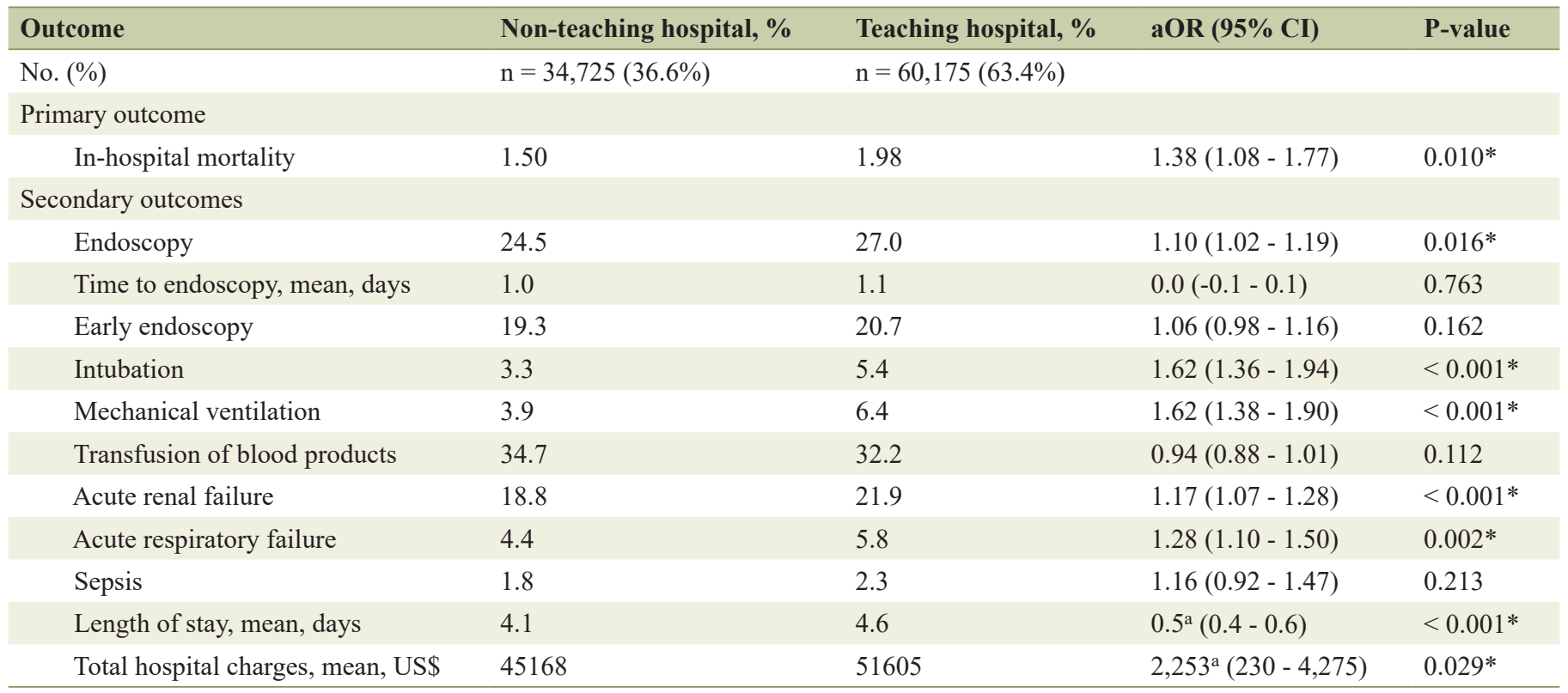

*Statistically significant. aAdjusted mean difference. aOR: adjusted odds ratio; Cl: confidence interval; NVUGIB: non-variceal upper gastrointestinal bleeding. 
both cohorts would have no difference in their need for RBC transfusion if they obtained endoscopy at similar times.

Our study has several strengths. Concerning our literature review, this is one of the few studies that has evaluated hospital outcomes of NVUGIB at teaching vs. non-teaching hospitals. Our use of the largest inpatient hospitalization database in the USA increases the power of our study. Our scientific questioning and analysis technique also contributes new information to a largely understudied topic of teaching hospital status effect on the outcomes on NVUGIB.

Our study is not without its limitations however, and the current limitations are as follows. The retrospective nature of our study establishes associations but cannot imply causality. In our study, we had a higher number of patients managed at teaching hospitals vs. non-teaching hospitals. The NIS reports data on hospitalization rather than individual patients, hence patients hospitalized on multiple occasions can be counted multiple times [27]. NIS does not account for the acuity or severity of the patient's condition on admission, hence we cannot determine if this may have affected our hospital outcomes [28]. NIS does not contain laboratory data, vital signs, or medication use and so we could not calculate pre-endoscopic risk scores, Glasgow or Blatchford scores. Our data do not have specific information on the involvement of fellows, the training or expertise of the fellows. We also could not identify specific interventions that were done during endoscopy. Finally, we were unable to obtain information on post-discharge follow-up in our cohort of patients.

\section{Conclusion}

Our study demonstrated that patients managed for NVUGIB at non-teaching hospitals had better in-hospital outcomes when compared to NVUGIB patients managed at teaching hospitals. Further research is encouraged to evaluate why patients at nonteaching hospitals fared better. Nonetheless, our findings contribute novel information on this topic.

\section{Supplementary Material}

Suppl 1. Used ICD-10 Codes

\section{Acknowledgments}

None to declare.

\section{Financial Disclosure}

None to declare.

\section{Conflict of Interest}

None to declare.

\section{Informed Consent}

Not applicable.

\section{Author Contributions}

Jennifer C. Asotibe MD, Hafeez Shaka MD, and Emmanuel Akuna MD are credited with substantial contributions to the drafting of the manuscript, interpretation of the data, design of the work, revision of intellectual content, and final approval of the submitted version of the manuscript. Niveda Shekar MD, Hassam Shah MD, and Marcelo Ramirez MD are credited with the drafting of the manuscript, literature review, and discussion of data. Syed Ali Amir Sherazi MD, and Katayoun Khoshbin MD are credited with the literature review and revision of intellectual content for the manuscript. Hemant Mutneja MD, and Bashar Attar MD are credited with critical revision of the entire manuscript, literature review, and final approval of the submitted manuscript.

\section{Data Availability}

The authors declare that data supporting the findings of this study are available within the article.

\section{Abbreviations}

NVUGIB: non-variceal upper gastrointestinal bleeding; UGIB: upper gastrointestinal bleeding; NIS: National Inpatient Sample; HCUP: Healthcare Cost and Utilization Project; LOS: length of stay; THC: total hospital charges; GI: gastrointestinal

\section{References}

1. Biecker E. Diagnosis and therapy of non-variceal upper gastrointestinal bleeding. World J Gastrointest Pharmacol Ther. 2015;6(4):172-182.

2. Gralnek IM, Dumonceau JM, Kuipers EJ, Lanas A, Sanders DS, Kurien M, Rotondano G, et al. Diagnosis and management of nonvariceal upper gastrointestinal hemorrhage: European Society of Gastrointestinal Endoscopy (ESGE) Guideline. Endoscopy. 2015;47(10):a1-46.

3. Lanas A, Dumonceau JM, Hunt RH, Fujishiro M, Scheiman JM, Gralnek IM, Campbell HE, et al. Non-variceal upper gastrointestinal bleeding. Nat Rev Dis Primers. 2018;4:18020.

4. Kyaw MH, Lau JYW. High rate of mortality more than 30 days after upper gastrointestinal bleeding. Clin Gastroenterol Hepatol. 2017;15(12):1858-1859.

5. Patel P, Rotundo L, Orosz E, Afridi F, Pyrsopoulos N. Hospital teaching status on the outcomes of patients with esophageal variceal bleeding in the United States. World J Hepatol. 2020;12(6):288-297.

6. Papanikolaou PN, Christidi GD, Ioannidis JP. Patient 
outcomes with teaching versus nonteaching healthcare: a systematic review. PLoS Med. 2006;3(9):e341.

7. Au AG, Padwal RS, Majumdar SR, McAlister FA. Patient outcomes in teaching versus nonteaching general internal medicine services: a systematic review and meta-analysis. Acad Med. 2014;89(3):517-523.

8. Shetty KD, Bhattacharya J. Changes in hospital mortality associated with residency work-hour regulations. Ann Intern Med. 2007;147(2):73-80.

9. Shelton J, Kummerow K, Phillips S, Arbogast PG, Griffin M, Holzman MD, Nealon W, et al. Patient safety in the era of the 80-hour workweek. J Surg Educ. 2014;71(4):551559.

10. Burke LG, Frakt AB, Khullar D, Orav EJ, Jha AK. Association between teaching status and mortality in US hospitals. JAMA. 2017;317(20):2105-2113.

11. Pioppo L, Bhurwal A, Reja D, Tawadros A, Mutneja H, Goel A, Patel A. Incidence of non-variceal upper gastrointestinal bleeding worsens outcomes with acute coronary syndrome: result of a national cohort. Dig Dis Sci. 2021;66(4):999-1008.

12. Kohn A, Ancona C, Belleudi V, Davoli M, Giglio L, Fusco D, Andreoli A, et al. The impact of endoscopy and specialist care on 30-day mortality among patients with acute non-variceal upper gastrointestinal hemorrhage: an Italian population-based study. Dig Liver Dis. 2010;42(9):629-634.

13. Schmidt MS, Preisler L, Fabricius R, Svenningsen P, Hillingso J, Svendsen LB, Sillesen M. Effect of hospital-admission volume on outcomes following acute nonvariceal upper gastrointestinal bleeding. Dan Med J. 2019;66(2):A5531.

14. Project HCaU. Introduction to the HCUP National Inpatient Sample (NIS). https://www.hcup-us.ahrq.gov/db/ nation/nis/NIS_Introduction_2016.jsp and https://www. hcup-us.ahrq.gov/db/nation/nis/NIS_Introduction_2017. jsp. Accessed January 20, 2021.

15. Moore BJ, White S, Washington R, Coenen N, Elixhauser A. Identifying increased risk of readmission and in-hospital mortality using hospital administrative data: the AHRQ Elixhauser Comorbidity Index. Med Care. 2017;55(7):698-705.

16. Ojemolon PE, Shaka H, Edigin E, Gomez TMA, Eseaton P, Bello J, Azubuike C, et al. Impact of diabetes mellitus on outcomes of patients with knee osteoarthritis who underwent knee arthroplasty: an analysis of the nationwide inpatient sample. Cureus. 2020;12(6):e8902.

17. Ayanian JZ, Weissman JS. Teaching hospitals and quality of care: a review of the literature. Milbank Q. 2002;80(3):569-593.

18. Rosenthal GE, Harper DL, Quinn LM, Cooper GS. Severity-adjusted mortality and length of stay in teaching and nonteaching hospitals. Results of a regional study. JAMA. 1997;278(6):485-490.

19. Shahian DM, Liu X, Meyer GS, Torchiana DF, Normand SL. Hospital teaching intensity and mortality for acute myocardial infarction, heart failure, and pneumonia. Med Care. 2014;52(1):38-46.

20. Yiadom M, Baugh CW, Barrett TW, Liu X, Storrow AB, Vogus TJ, Tiwari V, et al. Measuring Emergency Department Acuity. Acad Emerg Med. 2018;25(1):65-75.

21. Mueller SK, Lipsitz S, Hicks LS. Impact of hospital teaching intensity on quality of care and patient outcomes. Med Care. 2013;51(7):567-574.

22. Rich EC, Liebow M, Srinivasan M, Parish D, Wolliscroft JO, Fein O, Blaser R. Medicare financing of graduate medical education. J Gen Intern Med. 2002;17(4):283-292.

23. Fishman LE, Bentley JD. The evolution of support for safety-net hospitals. Health Aff (Millwood). 1997;16(4):30-47.

24. Werner RM, Kolstad JT, Stuart EA, Polsky D. The effect of pay-for-performance in hospitals: lessons for quality improvement. Health Aff (Millwood). 2011;30(4):690698.

25. Rosenthal MB, Frank RG, Li Z, Epstein AM. Early experience with pay-for-performance: from concept to practice. JAMA. 2005;294(14):1788-1793.

26. Singh H, Thomas EJ, Petersen LA, Studdert DM. Medical errors involving trainees: a study of closed malpractice claims from 5 insurers. Arch Intern Med. 2007;167(19):2030-2036.

27. Edigin E, Ojemolon PE, Eseaton PO, Jamal S, Shaka H, Akuna E, Asemota IR, et al. Systemic sclerosis is associated with increased inpatient mortality in patients admitted for acute coronary syndrome: analysis of the national inpatient sample. J Clin Rheumatol. 2020;12(8):e9799.

28. Edigin E, Asotibe J, Eseaton PO, Busari OA, Achebe I, Kichloo A, Jamal S, et al. Coexisting psoriasis is associated with an increased risk of hospitalization for patients with inflammatory bowel disease: an analysis of the National Inpatient Sample database. J Investig Med. 2021;69:857-862. 